\title{
Spectral signature of back reaction in correlated electron dynamics in intense electromagnetic fields
}

\author{
Alba de las Heras $\odot,{ }^{*}$ Carlos Hernández-García $\odot$, and Luis Plaja $\odot$ \\ Grupo de Investigación en Aplicaciones del Láser y Fotónica, Departamento de Física Aplicada, Universidad de Salamanca, \\ E-37008 Salamanca, Spain
}

(Received 25 February 2020; revised 5 May 2020; accepted 23 June 2020; published 9 July 2020)

\begin{abstract}
Few-electron atoms interacting with electromagnetic fields provide for privileged scenarios for disentangling elemental correlation mechanisms. We demonstrate that high-order harmonic generation (HHG) from neutral helium presents a distinctive trace of correlation back reaction in the electron dynamics prior to ionization. We identify a mechanism in which the field interacts with one of the electrons, while the second is excited to a Rydberg level through the Coulomb interaction. As back response to this correlated excitation, the first electron is knocked down to a lower level, from which it ionizes to generate high-order harmonics. Unlike other multielectron phenomena, where the electron-field interaction is modified by cross correlations with the rest of the interacting electrons, back reaction is a mechanism of autocorrelation. Our numerical simulations reveal back reaction as a relevant aspect in HHG from correlated two-electron systems, and paves the way to disentangle valence electron-electron correlation dynamics using high-harmonic spectroscopy.
\end{abstract}

DOI: 10.1103/PhysRevResearch.2.033047

\section{INTRODUCTION}

Correlation phenomena are at the foundations of complexity. They establish deep connections among the constituents of physical systems, giving rise to collective behaviors well beyond the reductionist point of view of independent particles. The Coulomb interaction between electrons determines the electric and magnetic properties of solids, being an essential ingredient in metal-insulator transitions, high-temperature superconductivity, and colossal magnetoresistance [1], but also affecting the exciton states in quantum wells [2] or the energy transport properties in carbon nanotubes [3]. Among the different correlation effects, back action represents the intriguing possibility of self-affected dynamics, as a response to the constituent's own modifications to a correlated environment. Correlation back action is known to have profound implications in quantum systems coupled with reservoirs [4] and in quantum measurements [5]. Illustrative examples are the damping of a mechanical oscillator when coupled to an optical cavity, resulting in self-sustained oscillations [4], or the enhancement of restoring forces in trapped particles [6].

Few-electron atoms and molecules offer an excellent playground for testing isolated valence electron correlation effects, due to the absence of core electrons. Multielectron interactions have been extensively studied in the context of double photoionization, which may result from three general mechanisms: the so-called two-step-one (TS1), where the second electron is knocked out from the atom by the interaction with

\footnotetext{
*albadelasheras@usal.es

Published by the American Physical Society under the terms of the Creative Commons Attribution 4.0 International license. Further distribution of this work must maintain attribution to the author(s) and the published article's title, journal citation, and DOI.
}

a previously ionized electron; the shake-off mechanism ( $\mathrm{SO}$ ), which describes the ionization of the second electron as a response to the sudden nuclear descreening, resulting from the fast ionization of the first one; and, finally, dynamical bound-state electron correlation (GSC). TS1 and SO result from final-state correlations, while GSC corresponds to correlations in the initial state, previous to ionization. In the highfrequency regime the relative weight of these three mechanisms is not well resolved [7]. However, for lower frequencies, previous works have demonstrated distinct signatures of TS1 and GSC. Bound-electron shake-up excitation by the interaction with an ionizing first electron was observed in the kinetic energy of the dissociation fragments of $\mathrm{D}_{2}^{+}$[8]. TS1 is also at the origin of the enhancement in the double ionization rates [9] and, recently, it has been identified as a main ingredient in the generation of high-frequency secondary plateaus in high-order harmonic generation (HHG) [10-12]. Initial-state correlation is, on the other hand, responsible for an extension of the cutoff frequency of the HHG spectrum [13], where the dynamical nuclei descreening effectively increases the ionization energy of the second electron. Noticeably, the spectral signatures of these correlation-based mechanisms are clearly distinct from the uncorrelated phenomena, either the single active electron emission from the neutral or cation species, or the simultaneous recombination of two electrons [14-17].

An additional aspect studied recently is correlation back action (CBA) in the shake-up photoemission of the helium atom: as the ionizing electron knocks the second electron to an excited cationic state, the field-induced dipole of this second reacts on the first electron, delaying its ionization by few tens of attoseconds [18]. While CBA is a secondary aspect in shake-up processes, to our knowledge, its role in GSC, where the proximity of the two electrons enhances correlation interactions, remains barely addressed.

High-order harmonic generation (HHG) represents a reliable mechanism to produce coherent attosecond pulses up 
to the extreme ultraviolet or even soft $\mathrm{x}$ rays [19-21]. In atoms or molecules, HHG is understood as a three-step process-tunnel ionization, acceleration, and recombination $[22,23]$ - in the electronic wave function dynamics driven by an intense laser field. In addition to its technological relevance to probe ultrafast phenomena with extremely high temporal resolution, the resulting spectrum, composed of high harmonic frequencies, encodes information about the electron dynamics, orbitals, and target structure, that can be revealed using attosecond spectroscopy [18,24-27] and high-harmonic spectroscopy [28-30]. The main phenomenology underlying HHG can be described considering a single active electron (SAE) [31] occupying the outermost valence orbital of the atom or molecule under study. However, multielectron effects can influence the ionization or recombination steps, leading to unequivocal signatures in the harmonic and photoionization spectra.

In this article, we demonstrate that back action in GSC has a distinctive signature in the HHG spectra: a secondary plateau, extending to frequencies above the single active electron cutoff. We show that this spectral feature arises from the HHG from single-excited two-electron bound states, where one of the electrons-which we denote as secondary-is knocked up to a Rydberg level by the Coulomb interaction with its partner-the primary electron-which is the one effectively interacting with the laser field. In correspondence with the Coulomb excitation of the secondary electron, CBA results in the knock-down of the primary electron to a deeper energy level, from which it tunnels out through the potential barrier, initiating the HHG process. Note that, in our case of study, the secondary plateau is emitted by the (excited) neutral atom. This differentiates the phenomenon from other two-electron processes, leading to secondary plateaus, in which correlations enhance the HHG emission from the cation [10-12,32-34] or result from nonsequential double recombination [14-17]. Also note that, in previous works reporting extended plateaus in HHG from neutral atoms, both electrons play an active role interacting with the laser field. In those cases, the cutoff frequency extension is explained by the dynamical nuclei descreening resulting from a field-induced excitation [35], or from the motion of the bound electron affecting the recolliding electron [13].

\section{THEORETICAL MODELS AND NUMERICAL METHOD}

We numerically integrate the one-dimensional timedependent Schrödinger equation (TDSE) of the helium atom, described by the Hamiltonian

$$
\hat{H}=\hat{T}_{1}+\hat{T}_{2}+\hat{V}_{1, N}+\hat{V}_{2, N}+\hat{V}_{1,2},
$$

$\hat{T}_{i}=\left[\hat{P}_{i}+e A(t) / c\right]^{2} / 2 m$ being the kinetic energy operator for the electron $i, P_{i}$ its canonical momentum, $A(t)$ the vector potential, $c$ the speed of light in vacuum, $m$ the mass of the electron, and $e$ the absolute value of the electron's charge. $\hat{V}_{i, j}=Z_{i j} / \sqrt{\left(x_{i}-x_{j}\right)^{2}+a_{i j}^{2}}$ are the Coulomb interaction potentials (labels $\{1,2, N\}$, for both electrons and the nucleus, respectively). We consider four different models: (i) two active electrons (TAE), the exact 1D description of the $\mathrm{He}$ atom, described by $\hat{H}$ in Eq. (1) with $Z_{1, N}=Z_{2, N}=-2$,
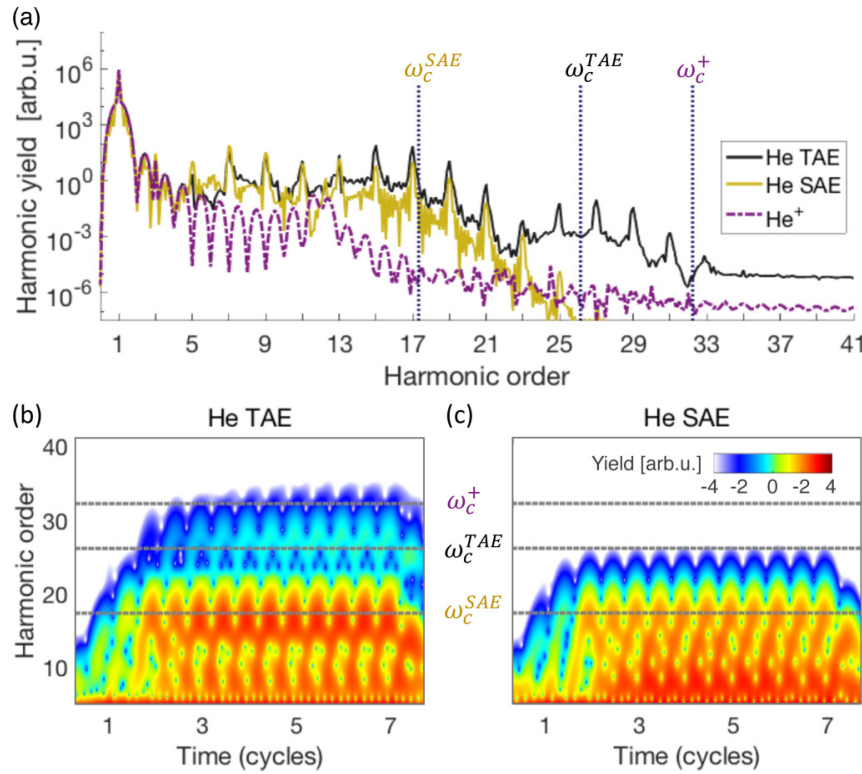

FIG. 1. (a) High-order harmonic spectrum from He considering two active electrons (He TAE, solid black line), a single active electron (He SAE, solid yellow line), and the exact description of $\mathrm{He}^{+}\left(\mathrm{He}^{+}\right.$, dash-dotted purple line). (b), (c) Time-frequency analysis of the HHG results from the TAE and SAE descriptions, respectively. The cutoff frequencies in the different cases are indicated with dotted lines. A high-energy secondary plateau emerges in the TAE calculations.

$Z_{1,2}=1, a_{1, N}=a_{2, N}=0.707$ a.u., $a_{1,2}=0.566$ a.u., so that the modeled ionization potentials of $\mathrm{He}$ and $\mathrm{He}^{+}$match the experimental values $I_{P}^{\mathrm{He}}=0.9$ a.u. and $I_{P}^{\mathrm{He}^{+}}=2.0$ a.u.; (ii) idle electron (IDL), considering the two correlated electronsprimary and secondary - yet the secondary disconnected from the laser field, i.e., $\hat{T}_{2}=\hat{P}_{2}^{2} / 2 m$, and the rest of the parameters in Eq. (1) as in TAE; (iii) single active electron (SAE), described by $\hat{H}$ with $\hat{T}_{2}=\hat{V}_{2, N}=\hat{V}_{1,2}=0$ and $a_{1, N}=$ 0.697 a.u.; and, finally, (iv) the exact 1D description of the helium cation $\left(\mathrm{He}^{+}\right)$, described by $\hat{H}$ with $\hat{T}_{2}=\hat{V}_{2, N}=\hat{V}_{1,2}=$ $0, a_{1, N}=0.707$ a.u., and $Z_{1, N}=-2$. The TDSE is solved in a $x_{1}, x_{2}$ grid (with spacing $\Delta x_{1}=\Delta x_{2}=0.16$ a.u. and box size $\left|x_{1_{\max }}\right|=\left|x_{2_{\max }}\right|=200$ a.u.) using the Crank-Nicolson method [36] with the split-operator approach to obtain the initial state via imaginary-time propagation [37] and to propagate it in real time with time step $\Delta t=0.02$ a.u. We implement a $\cos ^{1 / 8}$ mask function at the limits of the spatial box $(15 \%$ of the grid size) as an absorbing boundary. HHG spectra are computed as the squared modulus of the Fourier transform of the dipole acceleration. The driving field is modeled with an 8-cycle trapezoidal envelope ( 1 cycle of ramp-up, 6 cycles of constant amplitude, and 1 cycle of ramp-down), $515 \mathrm{~nm}$ central wavelength, and $1.6 \times 10^{14} \mathrm{~W} / \mathrm{cm}^{2}$ peak intensity. Additional results at other wavelengths, intensities, and pulse envelopes are shown in the Appendix.

\section{RESULTS AND DISCUSSION}

Figure 1(a) shows the comparison of the HHG spectrum from neutral He, within the TAE and SAE descriptions, as 


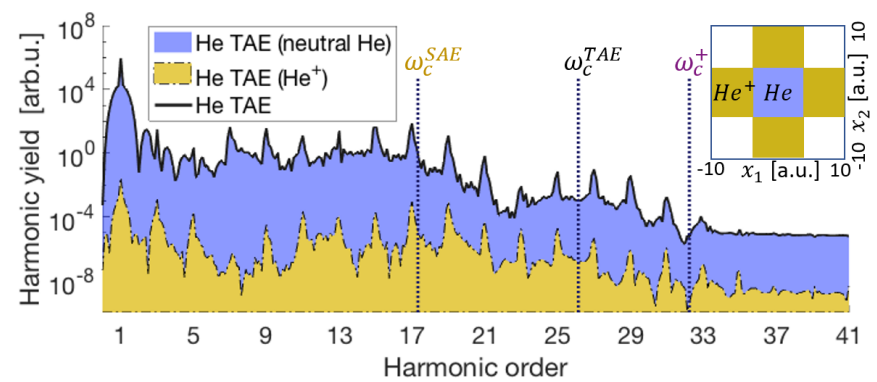

FIG. 2. Fraction of the high-harmonic emission from the two active electron (TAE) He, shown in Fig. 1(a), corresponding to the neutral $\mathrm{He}$ (blue area) and $\mathrm{He}^{+}$(yellow area) contributions. The total He TAE harmonic spectrum is shown for reference as a solid black line. Vertical dotted lines indicate the cutoff frequencies also shown in Fig. 1(a). The inset shows the regions of the two-electron coordinate space associated with the neutral atom (blue area) and the cation (yellow area).

well as from $\mathrm{He}^{+}$. Both models of neutral He show an excellent agreement up to the 23rd harmonic. In this region, the harmonic spectrum shows the typical nonperturbative plateau, extending up to the cutoff frequency, $\omega_{c}$. According to the standard description of HHG from a single active electron [31], the cutoff energy corresponds to [38]

$$
\hbar \omega_{c} \simeq 1.2 I_{P}+3.17 U_{P}
$$

$I_{P}$ being the ionization energy, and $U_{P}=e^{2} E_{0}^{2} / 4 m \omega_{0}^{2}$ the ponderomotive energy ( $E_{0}$ and $\omega_{0}$ are the driving electric field's amplitude and frequency, respectively). The cutoff frequency predicted by Eq. (2) considering the ionization potential of neutral $\mathrm{He}\left(\omega_{c}^{\mathrm{SAE}}\right)$ corresponds to the 17th harmonic. Noticeably, the TAE spectrum also includes a secondary plateau extending beyond the SAE cutoff frequency. Similar extensions have been reported before for higher intensities, resulting from the ionized fraction of He [39] or Ar [40]. However, Fig. 1(a) shows that the efficiency in HHG from $\mathrm{He}^{+}$is about five orders of magnitude lower. Note also that the cutoff frequency of the secondary plateau $\left(\omega_{c}^{\mathrm{TAE}}\right)$ is lower than that of the cation $\left(\omega_{c}^{+}\right)$.

In Figs. 1(b) and 1(c) we compare the time-frequency analysis of the HHG emission from the TAE and SAE descriptions, respectively. First, we observe the similar nature of the primary plateau in both descriptions, and the appearance of the secondary plateau in the TAE description. In addition, the efficiency of the emission of the secondary plateau increases progressively with time during the interaction. Hence, Fig. 1 demonstrates, on one hand, the multielectron nature of the process behind the extended spectrum and, on the other hand, the temporal delay in the buildup mechanism responsible for it. This latter property is a signature previously found in other correlation-mediated HHG pathways, other than shake-off emission. Such delays originate as the buildup of the correlation interchange typically requiring a previous excitation of one of the electrons [11].

Figure 2 displays further evidence of the secondary plateau being generated by the neutral atom, and not by the cation. We plot the contributions to the TAE harmonic spectrum shown in Fig. 1 of the different regions of the two-electron coordinate

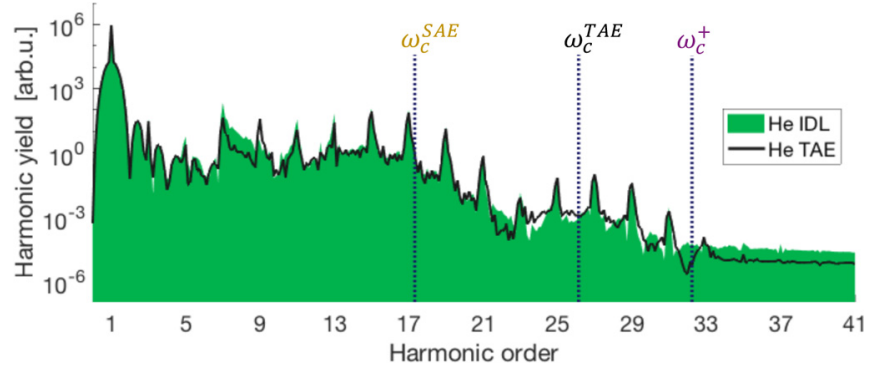

FIG. 3. High-order harmonic spectrum obtained from the idle electron model (He IDL, green area) compared to the exact twoelectron description (He TAE, black solid line). The cutoff frequencies are indicated with vertical dotted lines.

space (see inset in the figure). We consider the HHG emission from the wave function confined in two different spatial regions: $\left|x_{1}\right|<5$ a.u. while $\left|x_{2}\right|<5$ a.u., as the emission from the neutral He; and the disjoint spatial regions $\left(\left|x_{1}\right|<5\right.$ a.u. while $\left|x_{2}\right| \geqslant 5$ a.u.) and $\left(\left|x_{1}\right| \geqslant 5\right.$ a.u. while $\left.\left|x_{2}\right|<5\right)$, as the emission from the cation. According to these definitions, Fig. 2 shows that the TAE emission is dominated by the contribution of the neutral atom; i.e., the main HHG emission occurs when both electrons are close to the core. Therefore, the secondary plateau observed in the He TAE description is not due to a correlated-enhanced emission from the cation, as it is in [10-12], but from the neutral atom.

Let us focus our attention on the mechanism underlying the emission of the secondary plateau. As mentioned above, other correlation-induced HHG spectral extensions require both electrons interacting with the electromagnetic field, via TS1 or nuclei descreening. In these cases, one of the electrons is first excited - or ionized - by the electromagnetic field and, subsequently, the correlation interaction modifies the state of the second electron, which is then ionized to emit high harmonics. These mechanisms, however, do not apply in the present case. To demonstrate this, Fig. 3 shows the HHG spectrum resulting from the IDL model, in which only one of the two electrons is active- the primary one-interacting with the field, while the other one-the secondary-is artificially disconnected from the driving field. Remarkably, the highfrequency plateau is also present. This result unequivocally demonstrates that the multielectron dynamics responsible for the secondary plateau is only correlation-mediated.

In Fig. 4(a) we analyze the relative strength of the atomic dipole excitations during the interaction with the laser pulse, comparing the TAE and IDL models. The resonance peaks correspond to electronic transitions in neutral $\mathrm{He}$ (labeled with letters $\mathrm{A}, \mathrm{B}$, and $\mathrm{C}$ ), involving the energy levels depicted in Fig. 4(b). The A resonance peak points to the dominant excitation both in the TAE and IDL descriptions. The wave function corresponding to the excited state of the A transition, $\phi_{A}\left(x_{1}, x_{2}\right)$, is represented in the inset of Fig. 4(c). The density distribution reveals $\phi_{A}\left(x_{1}, x_{2}\right)$ as a single-excited state, with one ground electron near the nucleus and its partner occupying a Rydberg level. The energy level associated with $\phi_{A}$ is slightly lower than the ground level of $\mathrm{He}^{+}: \epsilon_{A}=\epsilon_{0}^{\mathrm{He}^{+}}+$ $\Delta \epsilon \simeq-2.22$ a.u., with $\Delta \epsilon=-0.22$ a.u. 

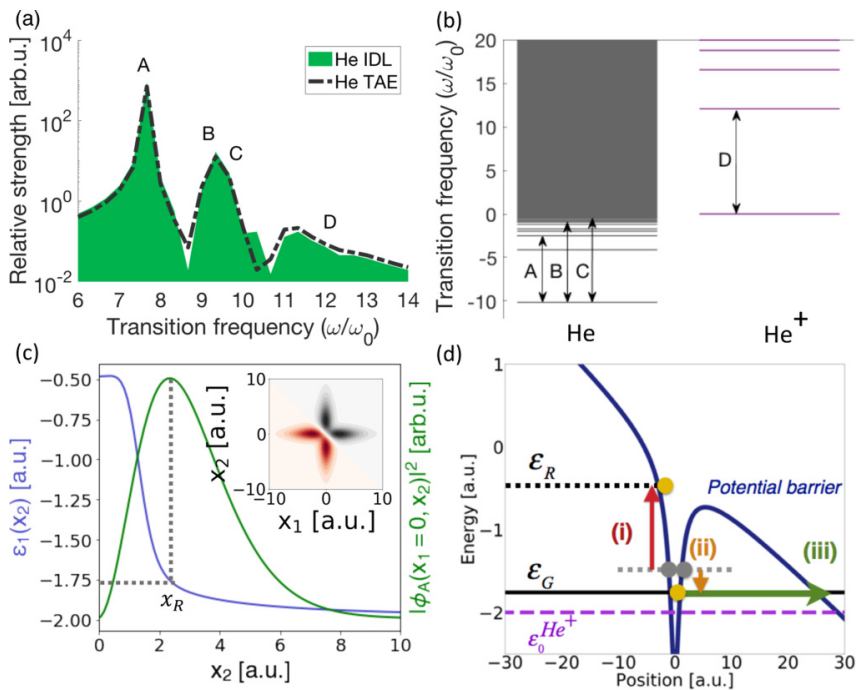

FIG. 4. (a) Relative strength of the atomic dipole excitations in the interaction with the laser pulse. (b) Energy levels of the neutral $\mathrm{He}$ and $\mathrm{He}^{+}$cation. (c) Blue line: Energy of the ground electron as a function of the position of the Rydberg electron; green line: probability density profile of electron 2 , assuming electron 1 located at $x_{1}=0,\left|\phi_{A}\left(0, x_{2}\right)\right|^{2}$. (d) Scheme of the correlated back-reaction mechanism previous to tunnel ionization: one electron is excited to a Rydberg level by correlation interaction (i), whereas the other is knocked down to a deeper level (ii). Finally this latter is ionized, leading to HHG (iii).

Let us draw some energy considerations from the information retrieved from state $\phi_{A}$. On one hand, the green line in Fig. 4(c) shows the probability density profile $\left|\phi_{A}\left(0, x_{2}\right)\right|^{2}$, i.e., assuming electron 1 in the ground state. The peak in the probability density points out the most probable radius, $x_{2}=$ $x_{R} \simeq 2.37$ a.u., of the Rydberg electron. On the other hand, the blue line in Fig. 4(c) shows the energy of the ground electron, 1 , as a function of the position of the Rydberg electron, 2, defined as

$$
\epsilon_{1}\left(x_{2}\right)=\frac{\int_{-\infty}^{\infty} \phi_{A}^{*}\left(x_{1}, x_{2}\right) \hat{H}_{1}\left(x_{1}, x_{2}\right) \phi_{A}\left(x_{1}, x_{2}\right) d x_{1}}{\int_{-\infty}^{\infty} \phi_{A}^{*}\left(x_{1}, x_{2}\right) \phi_{A}\left(x_{1}, x_{2}\right) d x_{1}}
$$

with $\hat{H}_{1}\left(x_{1}, x_{2}\right)=\hat{T}_{1}+\hat{V}_{1, N}+\hat{V}_{1,2} / 2$. At the Rydberg radius, $x_{2}=x_{R}$, the energy of the ground electron corresponds to $\epsilon_{G}=\epsilon_{1}\left(x_{R}\right) \simeq-1.76$ a.u. Consequently, the energy of the Rydberg electron can be estimated as $\epsilon_{R}=\epsilon_{A}-\epsilon_{G} \simeq$ -0.48 a.u., while the electron repulsion corresponds to $\epsilon_{e e}=$ $V_{1,2}\left(0, x_{R}\right) \simeq 0.41$ a.u. For the case in which the ground electron ionizes, leaving the Rydberg electron in the cation, the ionization potential corresponds to the difference between the initial state, $\epsilon_{i}=\epsilon_{A}$, and the final state after ionization $\epsilon_{f}=\epsilon_{R}-\epsilon_{e e} / 2$, i.e., the Rydberg level downshifted, since the electron-electron repulsion vanishes as the ground electron leaves the atom. Therefore, the ionization energy of the ground electron results in $I_{A}=\epsilon_{f}-\epsilon_{i} \simeq 1.55$ a.u. Inserting $I_{A}$ in Eq. (2), the cutoff harmonic order, for the parameters considered in Fig. 1, is $\simeq 26$, in perfect agreement with the cutoff frequency of the secondary plateau $\left(\omega_{c}^{\mathrm{TAE}}\right)$. This agreement [which can be better observed in Figs. 5(a) and 6] strongly suggests that the secondary plateau results from
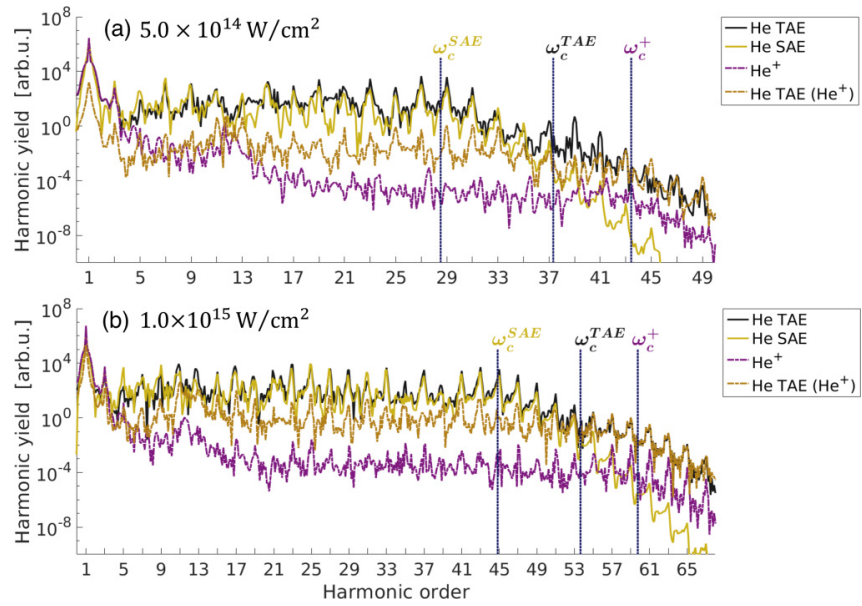

FIG. 5. High-order harmonic spectrum from He considering two active electrons (He TAE, solid black line), a single active electron (He SAE, solid yellow line), the SAE description of $\mathrm{He}^{+}\left(\mathrm{He}^{+}\right.$, dashed purple line), and the contribution from $\mathrm{He}^{+}$calculated in the TAE model (dashed orange line). We choose a central wavelength of $515 \mathrm{~nm}$ and two different peak intensities: (a) $5.0 \times 10^{14} \mathrm{~W} / \mathrm{cm}^{2}$ and (b) $1.0 \times 10^{15} \mathrm{~W} / \mathrm{cm}^{2}$.

HHG emitted by the ground electron, once it is ionized from the single-excited state $\phi_{A}$ of neutral He. Note that since the ground electron's ionization potential is lower than that of the He cation, the ionization rate is substantially higher, and thus the harmonic yield of the secondary plateau is considerably higher than the $\mathrm{He}^{+}$emission [see Fig. 1(a)].

In terms of correlation dynamics, due to the fact that the secondary plateau is also present in the IDL model, it becomes evident that the ground electron holds the primary-activerole interacting with the field, exciting the partner electron to a Rydberg level and, finally, generating the harmonic radiation. On the other hand, the IDL model demonstrates that the excitation of the Rydberg electron is due only to the Coulomb correlation. Interestingly enough, as back reaction, the excitation of the secondary electron lowers the energy level from which the primary electron is ionized. The modified ionization potential is, then, revealed by the value of the cutoff frequency of the secondary plateau.

The complete picture of the mechanism leading to the secondary plateau in HHG from He is summarized in Fig. 4(d): (i) the two-electron ground state of $\mathrm{He}$ is promoted to an electronic configuration where the primary electron remains close to the nucleus, and the secondary occupies a Rydberg state. The excitation of the secondary electron is mediated by electron-electron correlation. (ii) As a result of the correlation energy exchange, the Rydberg electron back-acts on the primary electron, lowering its energy. (iii) HHG takes place from the ionization of the primary electron in this modified ground level, giving rise to the emission of the harmonic secondary plateau. Further simulations for different driver's wavelenths, peak intensities, and pulse envelopes are provided in the Appendix showing the universality of this mechanism, and suggesting that visible driving wavelengths around $515 \mathrm{~nm}$, with moderate intensities around $1.6 \times 10^{14} \mathrm{~W} / \mathrm{cm}^{2}$, are optimal to observe the secondary plateau. 

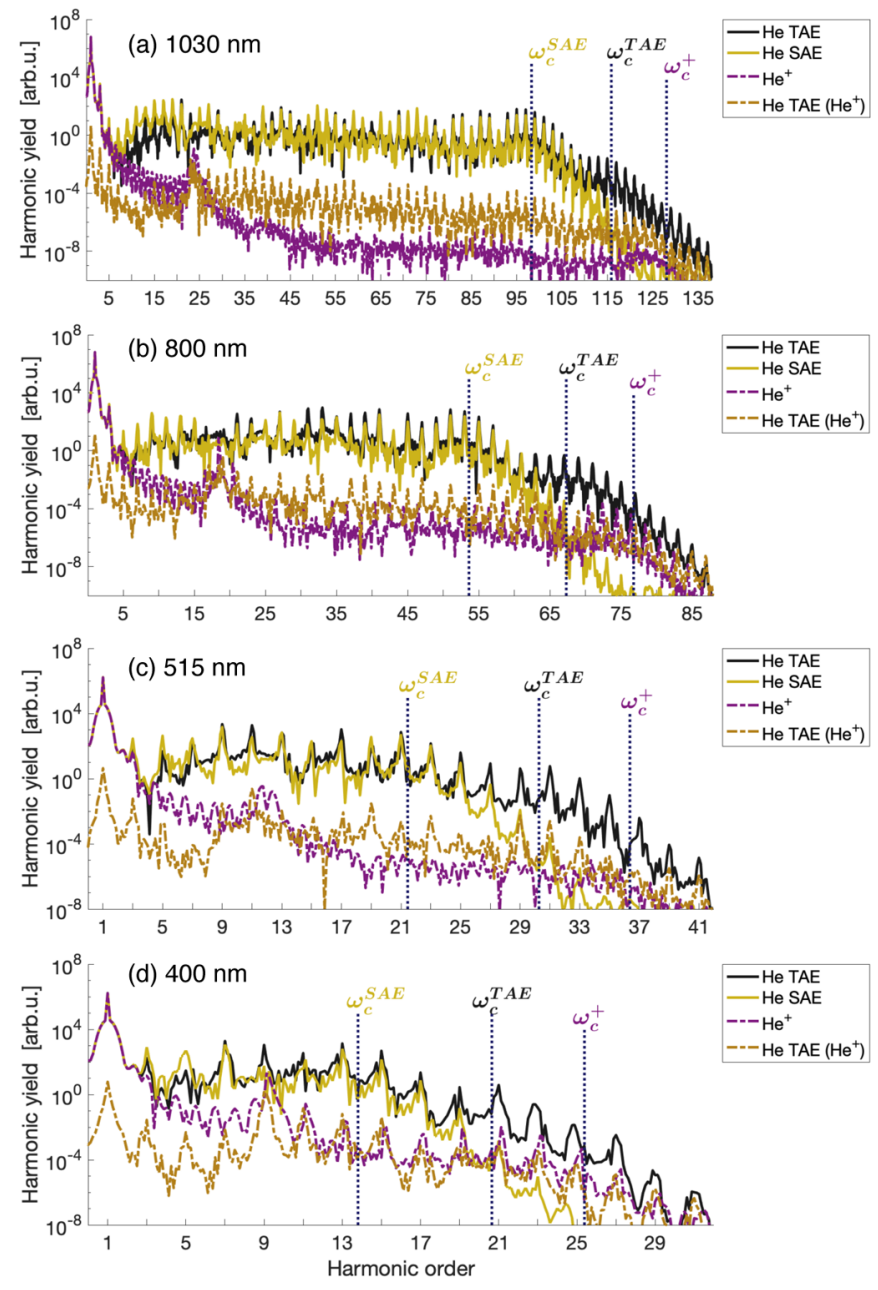

FIG. 6. High-order harmonic spectrum from He, considering two active electrons (He TAE, solid black line), a single active electron (He SAE, solid yellow line), the SAE description of $\mathrm{He}^{+}$ $\left(\mathrm{He}^{+}\right.$, dashed purple line), and the contribution from $\mathrm{He}^{+}$calculated in the TAE model (dashed orange line) at peak intensity $2.8 \times$ $10^{14} \mathrm{~W} / \mathrm{cm}^{2}$ and wavelengths (a) $1030 \mathrm{~nm}$, (b) $800 \mathrm{~nm}$, (c) $515 \mathrm{~nm}$, and (d) $400 \mathrm{~nm}$.

\section{CONCLUSIONS}

In conclusion, we have identified the spectral signature of a correlated back-action mechanism in the form of a secondary plateau in the high-harmonic spectrum from He. In contrast with other correlation-mediated mechanisms, one of the electrons evolves as effectively disconnected from the electromagnetic field, its dynamics resulting from the Coulomb correlation. The excitation of this secondary electron has a corresponding back action on the primary, lowering its energy. HHG from this modified level leads to the secondary highenergy plateau in the harmonic spectrum. Our work demonstrates that complex correlation interactions can be monitored using high-harmonic spectroscopy, and opens the possibility to study in other multielectron systems.

\section{ACKNOWLEDGMENTS}

We acknowledge fruitful discussions with Tenio Popmintchev. We also acknowledge support from Junta de
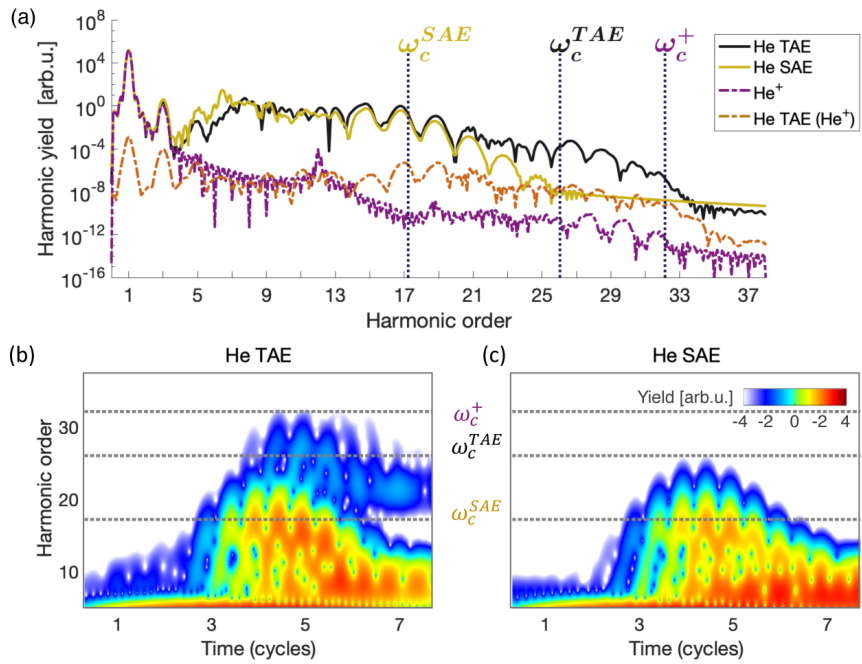

FIG. 7. (a) High-order harmonic spectrum from $\mathrm{He}$ in a driving field with a $\sin ^{2}$ envelope considering two active electrons (He TAE, solid black line), a single active electron (He SAE, solid yellow line), the SAE description of $\mathrm{He}^{+}\left(\mathrm{He}^{+}\right.$, dashed purple line), and the contribution from $\mathrm{He}^{+}$calculated in the TAE model (dashed orange line). Time-frequency analyses in TAE (b) and SAE (c) calculations are also shown.

Castilla y León FEDER funds (Project No. SA287P18) and Ministerio de Ciencia e Innovación (FIS2016-75652-P and PID2019-106910GB-I00). C.H.-G. acknowledges Ministerio de Ciencia, Innovación, y Universidades for a Ramón y Cajal contract (RYC-2017-22745), co-funded by the European Social Fund. This project has received funding from the European Research Council (ERC) under the European Union's Horizon 2020 research and innovation program (Grant Agreement No. 851201).

\section{APPENDIX: UNIVERSALITY OF THE BACK-ACTION SIGNATURE}

In this Appendix, we present the trace of correlated back action (CBA) in high-order harmonic generation (HHG) for different central wavelengths, peak intensities, and pulse envelopes of the driving field. Our simulations indicate that CBA is the relevant mechanism at low and moderate intensities, whereas the recollision-enhanced response of $\mathrm{He}^{+}$-reported before on HHG from Be [10], a two-electron molecular system [11], Li and $\mathrm{Na}$ [12]—dominates at high intensities, both forming a high-energy secondary structure.

In Fig. 5, we show the HHG spectra when irradiating at a central wavelength of $515 \mathrm{~nm}$ and two illustrative peak intensities: (a) $5.0 \times 10^{14} \mathrm{~W} / \mathrm{cm}^{2}$ and (b) $1.0 \times 10^{15} \mathrm{~W} / \mathrm{cm}^{2}$. The driving field is modeled with a trapezoidal envelope of 8 cycles ( 1 cycle of ramp-up, 6 cycles of constant amplitude, and 1 cycle of ramp-down). We have performed simulations within different theoretical models of neutral $\mathrm{He}$ - two active electrons (He TAE, solid black) and a single active electron (He SAE, dashed yellow) - and $\mathrm{He}^{+}$. For this latter case, we compute $\mathrm{HHG}$ from $\mathrm{He}^{+}$(dashed purple) or from the spatial region of TAE $\mathrm{He}$, corresponding to the $\mathrm{He}^{+}$ contribution (dashed orange; see Fig. 4). The high-energy 
secondary plateau originating from CBA is still visible at $5.0 \times 10^{14} \mathrm{~W} / \mathrm{cm}^{2}$ [mainly the 37th, 39th, and 41 st harmonic orders in Fig. 5(a)]. The calculated contributions of $\mathrm{He}^{+}$ are unable to explain the yield of the secondary plateau, because in the case of CBA the emission signature results from neutral He. As the intensity is increased [see Fig. 5(b)], the harmonic signal coming from the $\mathrm{He}^{+}$cation dominates over the signature of CBA.

Figure 6 shows the HHG spectra obtained for a peak intensity of $2.8 \times 10^{14} \mathrm{~W} / \mathrm{cm}^{2}$ and for central wavelengths of (a) $1030 \mathrm{~nm}$, (b) $800 \mathrm{~nm}$, (c) $515 \mathrm{~nm}$, and (d) $400 \mathrm{~nm}$. We consider the He TAE (solid black), He SAE (dashed yellow), and $\mathrm{He}^{+}$(dashed purple) models. Remarkably, for all the driving wavelengths considered, the secondary plateau is clearly observed. One can notice that the efficiency of the secondary plateau compared to the primary plateau increases as the wavelength decreases. However, for the shorter wavelengths, the contribution of the $\mathrm{He}^{+}$cation increases over the CBA signature, as the ionization probability is also higher. As a trade-off, the driving field of $515 \mathrm{~nm}$ represents a good landmark to observe this novel correlation mechanism.

Another interesting aspect is whether the back-reaction mechanism depends on the envelope of the laser pulse. In order to briefly explore this, we present in Fig. 7 the results for a driving field with a $\sin ^{2}$ envelope of 8 cycles, $515 \mathrm{~nm}$ of wavelength, and $1.6 \times 10^{14} \mathrm{~W} / \mathrm{cm}^{2}$ peak intensity. Again, the results in the main plateau (up to the 19th harmonic) are suitably reproduced in both the TAE and SAE descriptions, indicating a monoelectronic behavior. At higher frequencies, the high-harmonic emission only arises in the TAE calculation due to the multielectronic character of the back-reaction process. The cutoff of the secondary plateau matches the predicted value considering the ionization potential corresponding to the ground electron of the second excited two-electron state of He: $I_{A}=1.55$ a.u. This agreement implies that the fundamental scheme behind the CBA process is not altered depending on the form of the driving envelope. The TAE timefrequency analysis [Fig. 7(b)] corroborates the appearance of the secondary plateau, which is emitted in phase with the primary one. Moreover, during the first 3 cycles of the interaction, we observe a low-frequency emission (frequencies below the 15th harmonic) related to the fluorescence from single-excited states of neutral He. Since the atomic excitation is induced by electron correlation, this signal is absent in the SAE time-frequency analysis [Fig. 7(c)].

Finally, we would like to state that we do not foresee major complications to observe the secondary plateau when considering macroscopic phase matching, in comparison to the main SAE plateau. Macroscopic simulations involving harmonic phase matching for the TAE model are beyond the computational capabilities.
[1] Y. Tokura and N. Nagaosa, Science 288, 462 (2000).

[2] D. B. Turner and K. A. Nelson, Nature (London) 466, 1089 (2010).

[3] S. J. Tans, M. H. Devoret, R. J. Groeneveld, and C. Dekker, Nature (London) 394, 761 (1998).

[4] F. Sun, X. Dong, J. Zou, M. I. Dykman, and H. B. Chan, Nat. Commun. 7, 12694 (2016).

[5] T. P. Purdy, K. E. Grutter, K. Srinivasan, and J. M. Taylor, Science 356, 1265 (2017).

[6] M. L. Juan, R. Gordon, Y. Pang, F. Eftekhari, and R. Quidant, Nat. Phys. 5, 915 (2009).

[7] K.-i. Hino, T. Ishihara, F. Shimizu, N. Toshima, and J. H. McGuire, Phys. Rev. A 48, 1271 (1993).

[8] I. V. Litvinyuk, F. Légaré, P. W. Dooley, D. M. Villeneuve, P. B. Corkum, J.. Zanghellini, A. Pegarkov, C. Fabian, and T. Brabec, Phys. Rev. Lett. 94, 033003 (2005).

[9] B. Feuerstein, R. Moshammer, D. Fischer, A. Dorn, C. D. Schröter, J. Deipenwisch, J. R. Crespo Lopez-Urrutia, C. Höhr, P. Neumayer, J. Ullrich, H. Rottke, C. Trump, M. Wittmann, G. Korn, and W. Sandner, Phys. Rev. Lett. 87, 043003 (2001).

[10] I. Tikhomirov, T. Sato, and K. L. Ishikawa, Phys. Rev. Lett. 118, 203202 (2017).

[11] P. M. Abanador, F. Mauger, K. Lopata, M. B. Gaarde, and K. J. Schafer, Phys. Rev. A 97, 043414 (2018).

[12] Y. Li, T. Sato, and K. L. Ishikawa, Phys. Rev. A 99, 043401 (2019).

[13] J. Prager, S. X. Hu, and C. H. Keitel, Phys. Rev. A 64, 045402 (2001).

[14] P. Koval, F. Wilken, D. Bauer, and C. H. Keitel, Phys. Rev. Lett. 98, 043904 (2007).
[15] K. K. Hansen and L. B. Madsen, Phys. Rev. A 93, 053427 (2016).

[16] L. Feng, W. Li, M. Yuan, Y. Duan, and T. Chu, Phys. Lett. A 379, 805 (2015).

[17] A. Chacón, M. F. Ciappina, and M. Lewenstein, Phys. Rev. A 94, 043407 (2016).

[18] M. Ossiander, F. Siegrist, V. Shirvanyan, R. Pazourek, A. Sommer, T. Latka, A. Guggenmos, S. Nagele, J. Feist, J. Burgdörfer, R. Kienberger, and M. Schultze, Nat. Phys. 13, 280 (2017).

[19] T. Popmintchev, M. C. Chen, D. Popmintchev, P. Arpin, S. Brown, S. Ališauskas, G. Andriukaitis, T. Balčiunas, O. D. Mücke, A. Pugzlys, A. Baltuška, B. Shim, S. E. Schrauth, A. Gaeta, C. Hernández-García, L. Plaja, A. Becker, A. JaronBecker, M. M. Murnane, and H. C. Kapteyn, Science 336, 1287 (2012).

[20] P. Agostini and L. F. DiMauro, Rep. Prog. Phys. 67, 813 (2004).

[21] F. Krausz and M. Ivanov, Rev. Mod. Phys. 81, 163 (2009).

[22] K. J. Schafer, B. Yang, L. F. DiMauro, and K. C. Kulander, Phys. Rev. Lett. 70, 1599 (1993).

[23] P. B. Corkum, Phys. Rev. Lett. 71, 1994 (1993).

[24] M. Drescher, M. Hentschel, R. Kienberger, M. Uiberacker, V. Yakovlev, A. Scrinzi, T. Westerwalbesloh, U. Kleineberg, U. Heinzmann, and F. Krausz, Nature (London) 419, 803 (2002).

[25] M. Uiberacker, T. Uphues, M. Schultze, A. Verhoef, V. Yakovlev, M. Kling, J. Rauschenberger, N. Kabachnik, H. Schröder, M. Lezius, K. Kompa, H. Muller, M. Vrakking, S. Hendel, U. Kleineberg, U. Heinzmann, M. Drescher, and F. Krausz, Nature (London) 446, 627 (2007). 
[26] E. Goulielmakis, Z. Loh, A. Wirth, R. Santra, N. Rohringer, V. Yakovlev, S. Zherebtsov, T. Pfeifer, A. Azzeer, M. Kling, S. Leone, and F. Krausz, Nature (London) 466, 739 (2010).

[27] P. M. Kraus, M. Zurch, S. K. Cushing, D. M. Neumark, and S. R. Leone, Nat. Rev. Chem. 2, 82 (2018).

[28] O. Smirnova, Y. Mairesse, S. Patchkovskii, N. Dudovich, D. Villeneuve, P. Corkum, and M. Y. Ivanov, Nature (London) 460, 972 (2009).

[29] A. Shiner, B. Schmidt, C. Trallero-Herrero, H. J. Wörner, S. Patchkovskii, P. B. Corkum, J. Kieffer, F. Légaré, and D. Villeneuve, Nat. Phys. 7, 464 (2011).

[30] P. Peng, C. Marceau, and D. M. Villeneuve, Nat. Rev. Phys. 1, 144 (2019).

[31] K. C. Kulander, K. J. Schafer, and J. L. Krause, Int. J. Quantum Chem. 40, 415 (1991).

[32] N. Sarukura, K. Hata, T. Adachi, R. Nodomi, M. Watanabe, and S. Watanabe, Phys. Rev. A 43, 1669 (1991).
[33] D. G. Lappas, A. Sanpera, J. B. Watson, K. Burnett, P. L. Knight, R. Grobe, and J. H. Eberly, J. Phys. B: At., Mol. Opt. Phys. 29, L619 (1996).

[34] X.-M. Tong and S.-I. Chu, Phys. Rev. A 57, 452 (1998).

[35] N. Moiseyev and F. Weinhold, Phys. Rev. Lett. 78, 2100 (1997).

[36] J. Crank and P. Nicolson, Math. Proc. Cambridge Philos. Soc. 43, 50 (1947).

[37] R. Kosloff and H. Tal-Ezer, Chem. Phys. Lett. 127, 223 (1986).

[38] M. Lewenstein, P. Balcou, M. Y. Ivanov, A. L'Huillier, and P. B. Corkum, Phys. Rev. A 49, 2117 (1994).

[39] A. Sanpera, P. Jönsson, J. B. Watson, and K. Burnett, Phys. Rev. A 51, 3148 (1995).

[40] D. Popmintchev, C. Hernández-García, F. Dollar, C. Mancuso, J. A. Pérez-Hernández, M.-C. Chen, A. Hankla, X. Gao, B. Shim, A. L. Gaeta, M. Tarazkar, D. A. Romanov, R. J. Levis, J. A. Gaffney, M. Foord, S. B. Libby, A. Jaron-Becker, A. Becker, L. Plaja, M. M. Murnane, H. C. Kapteyn, and T. Popmintchev, Science 350, 1225 (2015). 